\title{
Linking Electronic Health Record and Trauma Registry Data: Assessing the Value of Probabilistic Linkage
}

\author{
Ashimiyu B. Durojaiye ${ }^{1,2}$ Lisa L. Puett ${ }^{3}$ Scott Levin ${ }^{4} \quad$ Matthew Toerper $^{5} \quad$ Nicolette M. McGeorge $^{1}$
} Kristen L. W. Webster ${ }^{1}$ Gurmehar S. Deol ${ }^{1,2}$ Hadi Kharrazi $^{2}$ Harold P. Lehmann ${ }^{2}$ Ayse P. Gurses ${ }^{1,2,6}$

${ }^{1}$ Center for Health Care Human Factors, Armstrong Institute for Patient Safety and Quality, Johns Hopkins Medicine, Johns Hopkins University, Baltimore, Maryland, United States

2 Division of Health Sciences Informatics, School of Medicine, Johns Hopkins University, Baltimore, Maryland, United States

${ }^{3}$ Department of Pediatric Nursing, Johns Hopkins Hospital, Johns Hopkins University School of Medicine, Johns Hopkins University, Baltimore, Maryland, United States

${ }^{4}$ Department of Emergency Medicine and Operations Integration, Whiting School of Engineering, Systems Institute, Johns Hopkins University School of Medicine, Johns Hopkins Hospital, Johns Hopkins University, Baltimore, Maryland, United States

${ }^{5}$ Department of Emergency Medicine and Operations Integration, Johns Hopkins University School of Medicine, Johns Hopkins Hospital, Johns Hopkins University, Baltimore, Maryland, United States

${ }^{6}$ Department of Anesthesiology and Critical Care Medicine and Health Policy and Management, Johns Hopkins University School of Medicine, Bloomberg School of Public Health, Malone Center for Engineering in Healthcare, Whiting School of Engineering, Johns Hopkins University, Baltimore, Maryland, United States

Methods Inf Med 2018;57:e3.

\section{Retraction Notice}

The publisher regrets an error that the above article was published online with an incorrect open access CC BY-NC-ND license.
Address for correspondence Ayse P. Gurses, PhD, MS, MPH, Department of Anesthesiology and Critical Care Medicine, Armstrong Institute Center for Health Care Human Factors, Johns Hopkins University, 750 East Pratt Street, 15th Floor, Baltimore, MD 21202, United States (e-mail: agurses1@jhmi.edu).

For this reason, the article has been retracted and the correct version of the article can be found here: https://doi. org/10.1055/s-0039-1681087. (c) 2018 Georg Thieme Verlag KG Stuttgart · New York 